\title{
Evaluation des périodes de vêlage des vaches locales des régions Maritime et des Plateaux au Sud-Togo
}

\author{
M. D. $\operatorname{KOTOE}^{1 *}, \mathrm{~K} \operatorname{SEME}^{1,2}$, K. A. KOSSOGA ${ }^{1}$, K. L. KOUMESSI ${ }^{1}$, W. PITALA ${ }^{2}$, \\ Y. $\mathrm{LOMBO}^{3}$ et K. KPEMOUA ${ }^{4}$
}

\author{
${ }^{1}$ Institut Togolais de Recherche Agronomique ; Centre de recherche Agronomique-zone Forestière \\ (ITRA/CRAF) Station d'Avétonou, BP 90 Kpalimé, Togo. \\ ${ }^{2}$ Ecole Supérieure d'Agronomie, Département des Sciences Animales et Vétérinaires, Université de Lomé \\ ESA/UL, BP 1515 Lomé, Togo. \\ ${ }^{3}$ Directeur Général de l'Institut Togolais de Recherche Agronomique (ITRA), BP 1163 Lomé, Togo. \\ ${ }^{4}$ Directeur Scientifique de la Recherche Agronomique, ITRA, Lomé, Togo. E-mail : itra@cafe.tg \\ *Auteur correspondant ; E- mail: kotmed@yahoo.fr / semejoseh@gmail.com; Tel: (00228) $90013404 /$ \\ 90163440
}

\section{RESUME}

La monte libre est le mode de reproduction pratiqué dans la plupart des élevages bovins au Togo. Ce système a pour conséquence une répartition naturelle de vêlage sur toute l'année. Cette étude a pour objectifs de faire l'état des lieux des périodes de vêlage des vaches sur une durée de cinq ans (2012 à 2016); d'évaluer son impact sur la fécondité des vaches et la viabilité des veaux afin de déterminer la période optimale pour une monte fécondante et efficiente. Pour se faire, des données ont été collectées sur 126 vaches de la station d'Avétonou, 61 vaches des élevages des préfectures d'Agou et 49 de l'Avé sur des races N'Dama et Locale de Type Somba. Les résultats ont montré qu'à la station d'Avétonou, il existe trois périodes de vêlage dont le principal est la période sèche (novembre à février) avec un taux de 79,68\% et une fréquence maximale de 14,94\% obtenue en décembre. Dans les autres élevages de l'étude, les mêmes observations ont été faites avec le même taux de vêlage et une fréquence maximale de $22,33 \%$ obtenue en novembre pour les élevages de la préfecture d'Agou et de 15,89\% comme fréquence maximale obtenue en décembre pour les élevages de la préfecture de l'Avé. La qualité alimentaire du pâturage qui survient le plus souvent en mars et avril constitue un facteur déterminant intervenant significativement dans les montes libres fécondantes des vaches. Par ailleurs, il n'existe pas de corrélation entre les périodes de vêlage et les mortalités des veaux, lesquelles sont reparties sur toute l'année. Ainsi, pour une bonne planification de la reproduction, mars et avril sont les mois indiqués pour des saillies fécondantes maximales.

(C) 2019 International Formulae Group. All rights reserved.

Mots clés : Monte libre, vêlage, fécondité, pâturage, mortalité, Togo.

\section{Evaluation of calving period of cows in the Maritime and Plateau Regions in southern Togo}

\section{ABSTRACT}

In most of the cattle farms in Togo, the reproduction system is based on free recovery. This system leads to a natural distribution of the calving period of the cows all over the year. The aim of this study is to make up the point of the calving period of the cows for a period of five (5) years 2012-2016; to evaluate the 
impact on the fecundity of the cows and the viability of the calves in order to determine the optimal period for an efficient fertile sally. For that matter, data has been collected on N'Dama, and the local breed named "Locale de Type Somba" from 126 cows in Avetonou station, 61 and 49 cows respectively in Agou and Ave prefecture farms. The results showed that in Avetonou station, there are three calving period among which the main is the dry season (November to February) with a mean rate of $79.68 \%$ and with a maximal frequency of $14.94 \%$ in December. In the other farms, the same observations have been noticed with the same calving rate and with a maximal frequency of $22.33 \%$ and $15.89 \%$ which occurred respectively in November in Agou prefecture farms and in December in Ave prefecture farms. The nutritional quality of the pasture which comes on mostly in march to April seems to be a determining factor which intervened significantly in the free recurring method of fecundation. Furthermore, there is no correlation between the calving period and the mortality of the calf's which is extended all over the year. Thus, March and April are the most favorable months for a maximal fecund sally in a reproduction program.

(C) 2019 International Formulae Group. All rights reserved.

Keywords: Free recovery, calving, fecundity, pasture, mortality, Togo.

\section{INTRODUCTION}

$\mathrm{Au}$ Togo, l'élevage des bovins contribue à $48 \%$ et $27 \%$ respectivement des besoins carnés et en lait (FAO, 2013). Cette faible production est due à: (i) la faible performance zoo sanitaire des élevages bovins (ii) l'insuffisance d'encadrement technique des éleveurs et (iii) la faible organisation de la reproduction (Adanlehoussi et Kotoé, 2005). Pour une approche de solution à cette faible production, une bonne maîtrise des paramètres de reproduction s'avère nécessaire en l'occurrence, les périodes et le contrôle de monte et de vêlage. Cependant, le système d'élevage au Togo est encore traditionnel et la saillie se fait de façon naturelle sans aucune intervention des techniciens dans la programmation de la reproduction ou dans la conduite de l'élevage. Il n'existe pas dans ces élevages de monte programmée ni d'insémination artificielle. Par conséquent, les périodes de gestation et de vêlage sont réparties de façon naturelle. Cette observation est faite dans $70 \%$ des troupeaux bovins togolais où les vêlages interviennent naturellement et exclusivement en période sèche (de novembre à février) (Seme, 2017). Cependant, dans certains troupeaux du SudTogo et en particulier à la station d'Avétonou, les mise-bas ont tendance à s'étendre sur toute l'année. Cette gestion naturelle de la reproduction constitue un paradoxe pour la filière laitière car les vêlages qui surviennent en saison sèche sont confrontées à des pénuries alimentaires dont les répercussions directes sont constatées sur le métabolisme de la vache d'une part, et sur la quantité et la qualité du lait, la nutrition du veau et la baisse du revenu de l'éleveur d'autre part. Cette étude a pour objectif de faire un état des lieux des périodes de mise-bas des vaches et d'identifier la période propice pour une monte fécondante et efficiente.

\section{MATERIEL ET METHODES Zone d'étude}

Cette étude a été conduite au SudTogo à la station de recherche d'Avétonou et dans des élevages privés des préfectures d'Agou (Etsi-Kopé et Togbossou-Kopé) et de l'Avé (Baka-Kopé) situées respectivement dans les régions Maritimes et des Plateaux.

Les deux régions jouissent d'un climat subéquatorial caractérisé par une grande saison de pluie allant de mars à juillet et une petite de septembre à octobre et une grande saison sèche qui s'étend de novembre à février et une petite qui couvre le mois d'août. La température moyenne varie entre $20^{\circ} \mathrm{C}$ et $35^{\circ}$ $\mathrm{C}$ et les précipitations oscillent entre $800 \mathrm{~mm}$ sur les côtes et $1600 \mathrm{~mm}$ à l'intérieur des régions. La végétation est constituée par un ensemble de savane arborée traversée par endroit de forêts galeries. Conformément à la biomasse du pâturage, trois saisons écologiques ont été déterminées correspondant aux stades végétatifs des pâturages : il s'agit de la saison 
sèche, de la saison post sécheresse, et de la saison des pluies (Tableau 2).

\section{Matériel animal}

Deux cent trente-six (236) vaches rustiques trypanotolérantes de races N'Dama et Locales de Types Somba (LTS) ont été le support de cette étude. Elles sont âgées de 4 à 12 ans et de rang de vêlage de 1 à 5 (Tableau $1)$.

La collecte des données a été faite grâce aux fiches de suivi de ces élevages et a couvert la période 2012 à 2016.

\section{Conduite du troupeau}

Les animaux sont conduits quotidiennement au pâturage sur parcours naturel constitué d'essence fourragère de Bracharia sp., de Stylosanthese sp., de Panicum sp., etc., de huit (8) heures à seize (16) heures. Le pâturage est annuellement victime de feux de végétation qui décime toute la végétation durant la saison sèche (novembre à février). La reprise du pâturage est marquée par trois stades végétatifs correspondant aux périodes écologiques de la zone (Tableau 2).

Une complémentation alimentaire est effectuée en saison sèche avec des graines de coton, des fanes d'arachide et de complément minéral. Le suivi sanitaire est assuré annuellement par trois traitements prophylactiques contre les helminthoses et les trypanosomoses. Les traitements contre les parasitoses externes sont réalisés mensuellement.

\section{Critères de choix des élevages}

Ces élevages ont été sélectionnés sur la base des critères suivants :

- leur proximité par rapport à la station d'Avétonou ;

- l'installation $\mathrm{du}$ troupeau au moins pendant cinq années ;

- l'élevage ayant au moins un effectif de cinquante (50) têtes avec dix (10) vaches naisseuses ;
- l'alimentation est essentiellement basée sur le pâturage ;

- la complémentation alimentaire constituée de paille de riz et autres résidus de récolte est au moins assurée en saison sèche;

- les géniteurs et les vaches naisseuses vivent ensembles pendant au moins cinq années ;

- l'existence des montes libres des vaches sans programmation pendant au moins cinq années.

\section{Analyses statistiques}

Les vêlages ont été classés par mois et par année pour chaque période écologique considérée. A partir du nombre de vêlage, les taux de mise-bas et les fréquences mensuelles de mise-bas ont été calculées (Formules 1 et 2):

Taux de vêlage $(\%)=$

$\frac{\text { Nombre de vêlage }}{\text { Effectif des femelles dans le troupeau }} \times 100$

Fréquence mensuelle de vêlage (\%) =

$\frac{\text { Nombre total de vêlage à un mois } t}{\text { Nombre total des mises bas durant les cinq années }} \times 100$

(2)

La répartition des vêlages a été évaluée par une analyse de variance au seuil de 5\% par le logiciel XLSTAT-Pro 7.1.

Les périodes de saillie fécondante ont été déterminées en se référant aux périodes de vêlage et à la durée de gestation qui est en moyenne de 9 mois pour les vaches.

La viabilité des veaux a été déterminée grâce aux informations collectées dans les registres portant sur les naissances et les mortalités des veaux âgés de 0 à 6 mois durant les cinq années d'étude. Le taux de mortalité des veaux a été calculé par la formule 3:

Taux de mortalité des veaux $(\%)=$ $\frac{\text { Nombre de veaux morts }}{\text { Nombre de veaux nés }} \quad x \quad 100$ 
Tableau 1: Facteurs considérés pour les vaches étudiées.

\begin{tabular}{|c|c|c|c|c|c|}
\hline \multirow{2}{*}{ Facteurs } & \multirow{2}{*}{ Paramètres } & \multirow{2}{*}{$\begin{array}{c}\text { Station } \\
\text { d'Avétonou }\end{array}$} & \multicolumn{2}{|c|}{ Préfecture Agou } & \multirow{2}{*}{$\begin{array}{c}\text { Préfecture Avé } \\
\text { Baka Kopé }\end{array}$} \\
\hline & & & Etsi Kopé & Togbossou Kopé & \\
\hline \multirow{3}{*}{ Races } & N'Dama (N) & 45 & 0 & 12 & 0 \\
\hline & Locale de Type & & & & \\
\hline & Somba (LTS) (N) & 81 & 35 & 14 & 49 \\
\hline \multirow{5}{*}{$\begin{array}{l}\text { Rang de } \\
\text { lactation }\end{array}$} & 1 & 14 & 5 & 3 & 3 \\
\hline & 2 & 22 & 13 & 9 & 20 \\
\hline & 3 & 51 & 8 & 7 & 13 \\
\hline & 4 & 27 & 6 & 3 & 9 \\
\hline & 5 & 12 & 3 & 4 & 4 \\
\hline
\end{tabular}

Tableau 2: Périodes écologiques et stades végétatifs des pâturages.

\begin{tabular}{llll}
\hline Saisons & Mois concernés & Stade végétatif du pâturage & $\begin{array}{l}\text { Pluviosité } \\
\text { mensuelle }(\mathbf{m m})\end{array}$ \\
\hline Sèche & novembre à février & Fourrage sec et peu abondant & 0 à 10 \\
\hline Post -sécheresse & mars à mai & Jeunes repousses d'herbes & 20 à 100 \\
\hline \multirow{2}{*}{ Pluie } & juin à octobre & $\begin{array}{l}\text { Fourrage en étape de floraison, } \\
\text { ligneux et gorgé d'eau. }\end{array}$ & 100 à 120 \\
\hline
\end{tabular}

\section{RESULTATS}

Taux moyen et fréquence mensuelle de vêlage

Le taux de vêlage varie de 78,29 à $79,68 \%$ dans les quatre (4) sites de l'expérience et la fréquence mensuelle maximale des vêlages se concentrent plus en période sèche surtout entre décembre et novembre (Tableaux 3 et 4 ).

\section{Périodes de saillie}

La période favorable de la saillie fécondante maximale est la période postsécheresse particulièrement mars et avril (Tableau 5).

\section{Viabilité des veaux}

Les mortalités des veaux âgés de 0 à 6 mois sont enregistrées sur toutes les périodes de l'année. Cependant, les mois de juillet et septembre paraissent plus vulnérables avec respectivement des taux de mortalité de $56,52 \%$ et $36,71 \%$ (Tableau 6 ). 
Tableau 3 : Taux de vêlage dans les élevages considérés.

\begin{tabular}{|c|c|c|c|c|c|}
\hline Paramètres étudiés & Avétonou & $\begin{array}{l}\text { Etsi } \\
\text { Kopé }\end{array}$ & $\begin{array}{l}\text { Togbossou } \\
\text { Kopé }\end{array}$ & $\begin{array}{l}\text { Baka } \\
\text { Kopé }\end{array}$ & Total \\
\hline Effectif des femelles & 126 & 35 & 26 & 49 & 236 \\
\hline $\begin{array}{l}\text { Nombre total de vêlage } \\
\text { durant les } 5 \text { années }\end{array}$ & 502 & 137 & 103 & 195 & 937 \\
\hline $\begin{array}{l}\text { Nombre moyen de } \\
\text { vêlage par année }\end{array}$ & 100 & 27 & 21 & 39 & 187 \\
\hline $\begin{array}{l}\text { Taux moyen de mise- } \\
\text { bas }(\%)\end{array}$ & 79,68 & 78,29 & 79,23 & 79,59 & 79,23 \\
\hline
\end{tabular}

Tableau 4 : Fréquences mensuelles des vêlages en fonction des périodes écologiques.

\begin{tabular}{|c|c|c|c|c|c|c|c|c|c|}
\hline \multirow{2}{*}{ Mois } & \multicolumn{2}{|c|}{ Station Avétonou } & \multicolumn{2}{|c|}{$\begin{array}{c}\text { Etsi } \\
\text { Kopé }\end{array}$} & \multicolumn{2}{|c|}{$\begin{array}{l}\text { Togbossou } \\
\text { Kopé }\end{array}$} & \multicolumn{2}{|c|}{$\begin{array}{l}\text { Baka } \\
\text { Kopé }\end{array}$} & \multirow{2}{*}{ Période } \\
\hline & $\begin{array}{l}\text { Nbre } \\
\text { MB }\end{array}$ & $\begin{array}{c}\text { Fréquence } \\
(\%)\end{array}$ & $\begin{array}{c}\text { Nbre } \\
\text { MB }\end{array}$ & $\begin{array}{c}\text { Fréquence } \\
(\%)\end{array}$ & $\begin{array}{c}\text { Nbre } \\
\text { MB }\end{array}$ & $\begin{array}{c}\text { Fréquence } \\
(\%)\end{array}$ & $\begin{array}{c}\text { Nbre } \\
\text { MB }\end{array}$ & $\begin{array}{c}\text { Fréquence } \\
(\%)\end{array}$ & \\
\hline Octobre & 28 & 5,58 & 12 & 8,76 & 32 & 31,06 & 18 & 9,29 & \multirow{5}{*}{ Sèche } \\
\hline Novembre & 64 & 12,75 & 15 & 10,95 & 23 & 22,33 & 27 & 13,84 & \\
\hline Décembre & 75 & 14,94 & 22 & 16,05 & 6 & 5,82 & 31 & 15,89 & \\
\hline Janvier & 53 & 10,56 & 14 & 10,22 & 4 & 3,88 & 25 & 12,82 & \\
\hline Février & 51 & 10,16 & 14 & 10,22 & 3 & 2,91 & 30 & 15,38 & \\
\hline Total1 & 271 & 53,99 & 77 & 56,16 & 68 & 66,00 & 131 & 67,22 & \\
\hline Mars & 45 & 8,96 & 14 & 10,21 & 4 & 3,88 & 29 & 14,87 & \multirow{3}{*}{$\begin{array}{c}\text { Post- } \\
\text { sècheresse }\end{array}$} \\
\hline Avril & 35 & 6,97 & 7 & 5,1 & 5 & 4,85 & 13 & 6,96 & \\
\hline Mai & 40 & 7,97 & 14 & 10,21 & 4 & 3,88 & 4 & 2,35 & \\
\hline Total2 & 120 & 23,90 & 35 & 25,52 & 13 & 12,61 & 46 & 24,18 & \\
\hline Juin & 25 & 4,98 & 7 & 5,21 & 6 & 5,87 & 3 & 1,99 & \multirow{4}{*}{ Pluvieuse } \\
\hline Juillet & 23 & 4,58 & 1 & 0,72 & 1 & 0,97 & 3 & 1,63 & \\
\hline Août & 27 & 5,38 & 10 & 7,29 & 7 & 6,79 & 8 & 4,98 & \\
\hline Septembre & 36 & 7,17 & 7 & 5,10 & 8 & 7,76 & 0 & & \\
\hline Total3 & 111 & 22,11 & 25 & 18,32 & 22 & 21,39 & 14 & 8,6 & \\
\hline $\mathrm{T} 1+2+3$ & 502 & 100 & 137 & 100 & 103 & 100 & 191 & 100 & \\
\hline
\end{tabular}


Tableau 5 : Périodes de saillie fécondante et fréquence de vêlage à Avétonou.

\begin{tabular}{|c|c|c|c|c|c|c|c|c|c|c|c|c|}
\hline $\begin{array}{l}\text { Périodes de } \\
\text { vêlage }\end{array}$ & Nov & Déc & Jan & Fév & Mar & Avril & Mai & Juin & Juil & Août & Sept & Oct \\
\hline $\begin{array}{l}\text { Fréquence } \\
\text { mensuelle de } \\
\text { vêlage }(\%)\end{array}$ & 12,75 & 14,94 & 10,56 & 10,16 & 8,96 & 6,97 & 7,97 & 4,98 & 4,58 & 5,38 & 7,17 & 5,58 \\
\hline \multirow{2}{*}{$\begin{array}{l}\text { Périodes de } \\
\text { saillie } \\
\text { fécondante }\end{array}$} & Mars & Avril & Mai & Juin & Juil & Août & Sept & Oct & Nov & Déc & Jan & Fév \\
\hline & & \multicolumn{2}{|c|}{ Favorable } & & \multicolumn{3}{|c|}{ Peu favorable } & \multicolumn{5}{|c|}{ Médiocre } \\
\hline
\end{tabular}

Tableau 6 : Viabilité des veaux nés par période (cas de la station d'Avétonou).

\begin{tabular}{|c|c|c|c|c|c|c|c|c|c|c|c|c|}
\hline Mois & Mars & Avril & Mai & Juin & Jlt & Août & Sept & Oct & Nov & Déc & Janv & Fév \\
\hline Veaux nés & 45 & 35 & 40 & 25 & 23 & 27 & 36 & 28 & 64 & 75 & 53 & 51 \\
\hline $\begin{array}{l}\text { Veaux } \\
\text { morts }\end{array}$ & 12 & 10 & 3 & 1 & 13 & 9 & 13 & 10 & 9 & 10 & 10 & 11 \\
\hline $\begin{array}{l}\text { Taux de } \\
\text { mortalité } \\
(\%)\end{array}$ & 26,67 & 28,57 & 7,50 & 4,00 & 56,52 & 33,33 & 36,11 & 35,71 & 14,06 & 13,33 & 18,87 & 21,57 \\
\hline Période & & Post-séc & heresse & & & Pluie & & & & écheress & & \\
\hline
\end{tabular}

\section{DISCUSSION}

Cette étude ressort qu'au Sud-Togo, les mise- bas sont diversement reparties sur toute l'année avec une dominance en saison sèche (octobre à février) et un pic en décembre. En effet, les fréquences de mise-bas enregistrées en saison sèche varient de 53,98 à $67,17 \%$ contre celles enregistrées en saison de pluie qui varient de 7,17 à $22,11 \%$. Ces résultats sont proches de ceux rapportés par Kouamo et al. (2009) sur la race N'Dama au Centre de Recherches Zootechniques de Minankro à Bouaké en Côte d'Ivoire où les mois de novembre à mars constituent les périodes de l'année pendant lesquelles $58 \%$ des mises bas sont enregistrées. Le taux de mise-bas de $79,68 \%$ obtenu à la station Avétonou est proche de ceux obtenus dans les autres élevages. Néanmoins, il reste supérieur à celui observé par Zampaligre et al. (2019) au Burkina-Faso qui est de 59,69\% mais plus proche de 70\% rapporté par d'Alkoiret et al. (2010). Ce taux correspond en moyenne au ratio de deux vêlages pour trois années consécutives et reste inférieur à un veau par an et par vache recommandé pour un élevage bovin ayant une bonne planification de la reproduction (Seme et al., 2016). De ce qui précède, la période favorable aux saillies fécondantes reste la période post sécheresse c'est-à-dire les mois de mars à mai. Meyer (2009) et Belkheri (2001) ont affirmé qu'en cas de monte naturelle libre, les saillies fécondantes correspondent le plus souvent au moment de l'année où la disponibilité alimentaire est la plus grande. La température en saison post-sécheresse $\left(25-28^{\circ} \mathrm{c}\right)$, les conditions d'élevage, en particulier la disponibilité du pâturage, seraient à l'origine des montes fécondantes enregistrées en ces périodes. En effet, les mois de mars à mai qui ont été marqués par des températures relativement fraiches, et l'arrivée des premières pluies de l'année ont favorisé les jeunes repousses de Panicum sp., de Bracharia sp. et de Stylosanthes sp., etc., après le passage de feu de végétation. Ainsi, les conditions atmosphériques combinées au 
pâturage qui a été amélioré en quantité et en qualité ont favorisé une augmentation de poids et un déclenchement de l'œstrus chez les vaches (Santos et al., 2009 ). Le pâturage a donc fortement influencé les périodes de saillie fécondante de mars et mai ce qui a permis les vêlages correspondants pendant la période sèche. Les vêlages et les saillies fécondantes, constituent donc des indicateurs physiologiques fortement influencés par l'alimentation et la température les quelles ont des répercussions directes sur le métabolisme et la fertilité des vaches. (Indetie et al., 2015). Ainsi, les vaches qui ont vêlé au cours des périodes sèches ont connu leur œstrus en période post-sécheresse. Au-delà de cette période, le pâturage se retrouve à l'étape de floraison entre juillet et septembre, période au cours de laquelle la qualité nutritionnelle du fourrage est dégradée; ce qui a pour corollaire, la réduction de la fertilité des vaches. (Blanc et al., 2004). En effet, la période de pluie (juillet à septembre) a été caractérisée par un fourrage abondant mais ligneux et gorgé d'eau; (Tableau 2); ce fourrage n'est pas de bonne qualité et n'a pas favorisé une bonne fertilité des vaches. ; c'est ce qui explique la faible fréquence de vêlage pour les vaches qui ont été mises en reproduction en ces périodes. Les auteurs Obese et al. (2010); Lopez et al. (2004) ont rapporté qu'une alimentation inadéquate est source de retard de croissance, une prolongation de l'intervalle entre le postpartum et la reprise du cycle, une augmentation de l'incidence de chaleur silencieuse, une réduction du taux de gestation, et une diminution du poids du veau à la naissance. Nous constatons ici que la reproduction (la fréquence mensuelle des vêlages) des vaches est influencée par les périodes écologiques de l'année. En effet, si le pic de vêlage est observé en novembre et décembre cela veut dire que la période de monte a été réalisée en mars et mai qui sont des périodes de repousse du pâturage. Cependant, certains auteurs ont affirmé que d'autres facteurs en dehors de l'alimentation peuvent influencer la fertilité des vaches. Meyer (2009) a démontré qu'en dehors de l'alimentation (pâturage), la fertilité des vaches en milieu tropical dépend de la photopériode, de l'intensité lumineuse, de la température ambiante et de la pluviométrie. Indetie et al. (2015), ayant travaillé sur les vaches de race friesian et sahiwal au Kénya, ont révélé que l'effet race est plus important que l'effet nutritionnel pour la production, pour la composition du lait et pour la performance de reproduction.

L'étude portant sur la mortalité des veaux a révélé que les mortalités sont enregistrées en tout moment de l'année. La mortalité des veaux n'est donc pas forcement liée à la période de vêlage. Néanmoins, les mois de juillet et septembre (période de pluie) paraissent plus vulnérables pour les veaux avec des taux respectifs de $56,52 \%$ et $36,11 \%$. $\mathrm{Au}$ regard de ces résultats, nous admettons que la période de pluie n'est pas la cause principale des mortalités des veaux mais constitue plutôt un facteur de risque pour la viabilité des veaux. En effet la diarrhée des veaux a des origines multiples (Maes, 2010). Zampaligre et al. (2019) ont rapporté que les mortalités dans les troupeaux juvéniles dans la zone péri-urbaine de Bobo-Dioulasso sont dues le plus souvent au déficit nutritionnel en saison sèche mais aussi au parasitisme pendant l'hivernage. Pour Youssao et al. (2013) cité par Zampaligre, la sousalimentation, l'absence de prophylaxie sanitaire appropriée et médicales sont les principales causes de mortalité des veaux en élevage traditionnel notamment pasteurs et agropasteurs.

Ainsi, pour sauvegarder la vie des veaux et accroître la fertilité des vaches, deux périodes se dégagent: une favorable à la saillie fécondante des vaches (début de la saison des pluies) et une favorable à la survie des veaux (saison sèche) (Meyer, 2009).

\section{Conclusion}

L'évaluation des périodes de mise-bas des vaches au sud du Togo est d'une grande importance dans l'amélioration de la reproduction des vaches. Pour ce faire, une bonne maitrise de la fécondité des vaches, des périodes de mise-bas, et une bonne planification des périodes de fécondation en 
cohérence avec l'abondance et la qualité du pâturage est nécessaire. Ces éléments constituent donc des facteurs déterminants dans le système de reproduction où, la majorité des vaches vêlent en saison sèche et la monte libre fécondante sont fortement liés à la qualité du pâturage. Ainsi, au sud du Togo où le système d'élevage est de type extensif et sans programmation de la reproduction, les mois de mars à mai sont les mieux indiqués pour des saillies fécondantes maximales. Cependant, les périodes post anœstrus après le vêlage doivent être entretenues par une alimentation complémentaire suffisante.

\section{CONFLIT D'INTERETS} les auteurs.

Il n'y a pas de conflit d'intérêts parmi

\section{CONTRIBUTIONS DES AUTEURS}

MDK a conçu le protocole, a participé à la collecte les données, a effectué les analyses statistiques et a participé à la rédaction du manuscrit. KS a contribué à la rédaction du protocole, aux traitements informatiques des données collectées, à la lecture et à la correction du manuscrit. KAK a participé à la recherche des informations dans le registre des vêlages sur les animaux de la station et à l'enregistrement des données. Il a participé à la lecture du manuscrit. KLK a participé à la collecte des données dans les troupeaux et la lecture du manuscrit. WP a participé à la lecture et à la correction du manuscrit. YL a facilité la formation en rédaction scientifique et a permis l'acquisition des outils nécessaires pour la collecte et l'analyse des données informatiques. KK a participé au suivi et à l'encadrement scientifiquement les travaux.

\section{REMERCIEMENTS}

Les auteurs de cet article tiennent à remercier les propriétaires des fermes, les techniciens de la station et les bouviers pour leur acceptation et leur disponibilité pour cette expérimentation. Nous remercions également le Directeur Général de l'ITRA et le Directeur du CRAF pour leurs apports logistiques et leurs conseils pour la réussite des travaux.

\section{REFERENCES}

Adanléhoussi A, Kotoe M. 2005. Elevage bovin. In Productions animales au Togo: Situation de référence. Institut Togolais de Recherche Agronomique (ITRA) Togo, 127p.

Alkoiret TI, Awohouedji DYG, Yacoubou AM. 2010. Paramètres démographiques des cheptels de bovins Borgou et N'Dama à la ferme d'élevage de l'Okpara au nord-est du Bénin. Int. J. Biol. Chem. Sci., 4(5): 1657-1666. DOI: http://dx.doi.org/10.4314/ijbcs.v4i5.6558 0

Belkheri B. 2001. Contribution à l'étude physiopathologique du post-partum chez la vache laitière. Thèse de magistrat INA, Alger, 99p.

Blanc F, Bocquier F, Debus N, Agabriel J, D'Hour P, Chilliard Y. 2004. La pérennité et la durabilité des élevages des ruminants dépendent des capacités adaptatives des femelles. INRAProductions Animales, 17(4) : 287-302.

Boma S, Nuto Y, Dayo GK, Bonfo B, N'Feide T. 2018. Caractérisation morpho-biométrique des populations bovines locales sans bosse au Togo. Int. J. Biol. Chem. Sci., 12(1) : 431-445. DOI : 10.4314/ijbcs.v12i1.34

CIRAD. 2002. Mémento de l'Agronome (éd). Cirad/Gret/Ministère des affaires Etrangères : Paris, France.

FAO. 2013. $4^{\text {ème }}$ Recensement National de l'Agriculture. Ministère de l'Agriculture, de L'Elevage et de la Pêche (MAEP), Direction des Statistiques Agricoles, de l'Informatique et de la Documentation (DSID), document définitif, 51p.

Ghozlane F, Yakhlef H, Yaici S. 2002. Performance de reproduction et de production laitière dans les bovins laitiers en Algérie. Anale de l'Institut National Agronomique El-Harrach, 24(1-2): p.63.

Indetie D, Musalia L, Bebe B, Wathuta $\mathrm{E}$, Indetie A, Kinywa J, Lukibisi F. 2015. Effect of body weight and conditions changes after parturition in the reproductive performance of sahiwal and 
friesian cattle. Bull. Anim. Hlth. Prd. Afr. AnGR.,166-173.

Indetie D, Musalia L, Bebe B, Wathuta E, Indetie A, Kinywa J, Lukibisi F. 2015. Effect of nutritional and supplementatiog and genotype on mik production and fertility of lactating dairy cattle under tropical conditions. Bull. Anim. Hlth. Prd. Afr. AnGR., 179- 190

Kouamo J, Sow A, Leye A, Sawadogo GJ, Ouedraogo GA. 2009. Amélioration des performances de production et de reproduction des bovins par l'utilisation del'insémination en Afrique Subsaharienne et au Sénégal en particulier : état des lieux et perspectives. RASPA., 7(3-4): 139-148.

Lopez H, Satler LD, Wiltbank MC. 2004. Relationship between level of milk production and estrus behavior of lactating dairy cow. Animal Production science, 81: 200-223.

Mae P. 2010. Ethiologie des diarrhées néonatale et transfert colostrale chez le veau : enquête dans la creuse. Thèse de doctorat vétérinaire, Ecole nationale d'Alford, $124 \mathrm{p}$.

Meyer C. 2009. Les variations saisonnières de la reproduction des bovins domestiques en zone tropicale Synthèse. Système d'élevage et produits animaux, Dep., Environnement et Société, Cirad TA, 17 p.

Obese FY, Dafour KA, Gomda Y, Bekoe E. 2010. Reproductive performance following artificial insemination in Sanga and crossbreed (Friesian x Sanga) cows in the Accra Plain of Ghana. In:
Sustainable improvement of animal Production and Health (Eds), Odongo NE, M, Viljoen GJ, IAEA Publication : Vienne, Austria ; 201-203.

Santos JP, Rutigliano HM, Sa Filho MF. 2009. Risk factors for resumption of postpartum estrus cycles and embryonic survival in lactating dairy cows. Animal Production Science, 110 : 207-221.

Seme K, Pitala W, Kulo EA, Zongo M, Boly H, Gbeassor M. 2016. Etude des conditions d'élevage des bovins laitiers et des possibilités d'introduction de l'insémination artificielle au Togo. RASPA, 14(1-2) : 23-29.

Seme K. 2017. Amélioration de la fécondité bovine par l'insémination artificielle au sud du Togo. Thèse de doctorat unique à l'école supérieur d'agronomie, université de Lomé, Togo, 161p.

Youssao AKI, Dahouda M, Attakpa EY, Koutinhouin GB, Ahounou GS, Toléba SS, Balogoun BS. 2013. Diversité des systèmes d'élevage des bovins de race bovine Borgou dans la Zone soudanienne du Bénin. Int. J. Biol. Chem. Sci., 7(1) :125-146. DOI : http://dx.doi.org/10 4314ijbcs.v7.ili.//

Zampaligre N, Savadogo M, Sangaré M. 2019. Analyse des paramètres démographiques et zootechniques du cheptel des élevages péri-urbain laitiers de la ville de Bobo-Dioulasso à l'ouest du Burkina-Faso. Int. J. Biol. Chem. Sci., 13(1): $141-151 . \quad$ DOI : http://dx.doi.org/10.4314/ijbcs.v13i1.35 